IJMMS 25:10 (2001) 669-677

PII. S0161171201003192

http://ijmms.hindawi.com

(c) Hindawi Publishing Corp.

\title{
ON THE EXISTENCE OF SOLUTION OF A TWO-POINTBOUNDARY VALUE PROBLEM IN A CYLINDRICAL FLOATING ZONE
}

\author{
SHI YONGDONG and DU LIANGSHENG
}

(Received 19 February 1999)

\begin{abstract}
Existence of one solution for a two-point boundary value problem with a positive parameter $Q$ arising in the study of surface-tension-induced flows of a liquid metal or semiconductor is studied. On the basis of the upper-lower solution method and Schauder's fixed point theorem, it is proved that the problem admits a solution when $0 \leq Q \leq 12.683$. This improves a recent result where $0 \leq Q<1$.
\end{abstract}

2000 Mathematics Subject Classification. Primary 34B10.

1. Introduction. Consider the following nonautonomous two-point boundary value problem (BVP) on $[0,1]$ :

$$
\begin{aligned}
& {\left[x\left(\frac{f^{\prime}}{x}\right)^{\prime}\right]^{\prime}+Q\left[f\left(\frac{f^{\prime}}{x}\right)^{\prime}-x\left(\frac{f^{\prime}}{x}\right)^{2}\right]=\beta x,} \\
& f(0)=f(1)=\left.\left(\frac{f^{\prime}}{x}\right)^{\prime}\right|_{x=0}=\left.\left(\frac{f^{\prime}}{x}\right)^{\prime}\right|_{x=1}-1=0,
\end{aligned}
$$

where $^{\prime}=d / d x$. This problem arises in the study of surface-tension induced flows of a liquid metal or semiconductor in a cylindrical floating zone of length $2 L$ and radius $R$. In dimensionless coordinates $(r, y)$, points of the cylinder are given by $-1 \leq y=$ $Y / L \leq 1,0 \leq x=r / R \leq 1$, with free surface $x=1$. The $(y, r)$-components of dimensionless velocity $(u, v)$ are, respectively, $u=2 A^{3}(\mathrm{Re}) f / x$ and $v=-2 A^{3}(\mathrm{Re}) f^{\prime} / x$, where Re is the Reynolds number $\left(Q=2 A^{3} \mathrm{Re}\right), A=L / R$ is the aspect ratio and $\beta$ is a constant to be determined. Assuming that the dimensionless pressure $p$ is a quadratic function of $y$, we find that the $r$-component of the acceleration equation in the Navier-Stokes energy system describing the flow of fluid and its temperature in the cylinder becomes (1.1a). The physical boundary conditions reduce to the conditions (1.1b) if we make the assumption that the free boundary is time-independent but not "flat."

Numerical solutions of (1.1) have been found [3] for $0 \leq Q \leq 32.7$ and $Q \geq 1749$. However, a theoretical proof on the existence of solutions of (1.1) has been done only for $0 \leq Q<1$ in [4]. Hence there is still a large gap between numerical experiments and theoretical results. In the present paper, on the basis of the upper-lower solution method and Schauder's fixed point theorem, we prove the existence of solutions for (1.1) with $0 \leq Q \leq 12.683$. Thereby we make a greater improvement of the existing results [4]. 
Our main result is the following theorem.

THEOREM 1.1. For $0 \leq Q \leq 12.683$, there exists a constant $\beta$ such that (1.1) admits a solution $f=f(x)$ satisfying on $(0,1)$

$$
-0.0406 \leq f(x) \leq 0, \quad-0.0935 \leq f^{\prime}(x) \leq \frac{1}{3}, \quad-0.43 \leq f^{\prime \prime}(x) \leq \frac{4}{3} .
$$

2. A technical treatment of (1.1). We observe that in (1.1), equation (1.1a) is a third order equation with an unknown constant $\beta$, while the boundary value condition (1.1b) contains four equalities. Hence following [4], we make the following technical treatment of (1.1).

Differentiating (1.1a) with respect to $x$, we obtain

$$
\begin{gathered}
{\left[\left(\frac{f^{\prime}}{x}\right)^{\prime}\right]^{\prime \prime}+\left[\frac{1+Q f}{x}\right]\left(\frac{f^{\prime}}{x}\right)^{\prime \prime}-\left[\frac{1+Q(x f)^{\prime}}{x^{2}}\right]\left(\frac{f^{\prime}}{x}\right)^{\prime}=0,} \\
f(0)=f(1)=\left.\left(\frac{f^{\prime}}{x}\right)^{\prime}\right|_{x=0}=\left.\left(\frac{f^{\prime}}{x}\right)^{\prime}\right|_{x=1}-1=0 .
\end{gathered}
$$

Let $\left(f^{\prime} / x\right)^{\prime}=g$. Then (2.1) has the following form:

$$
\begin{gathered}
g^{\prime \prime}+\left[\frac{1+Q f}{x}\right] g^{\prime}-\left[\frac{1+Q(x f)^{\prime}}{x^{2}}\right] g=0, \\
g(0)=g(1)-1=0 .
\end{gathered}
$$

To prove the existence of solutions for (1.1), we reduce to finding a fixed point problem. On the basis of the differential inequality technique to construct upper and lower solutions of (2.2), we consider the following set:

$$
D=\left\{f \mid f \in C^{1}[0,1], f(0)=f(1)=0, h(x) \leq f(x) \leq 0, m(x) \leq f^{\prime}(x) \leq n(x)\right\},
$$

where

$$
\begin{gathered}
h(x)=\frac{25}{119} x^{2}\left(x^{7 / 5}-1\right), \quad n(x)=\frac{1}{3} x^{4}, \\
m(x)=5 x\left(\frac{1}{26} x^{26 / 5}-\frac{1}{17} x^{17 / 5}+\frac{1}{7} x^{7 / 5}-\frac{10}{119}\right) .
\end{gathered}
$$

For any $f \in D$, if (1) equation (2.2) has a unique solution $g(x)$; and (2) the problem

$$
\begin{gathered}
\left(\frac{f^{*^{\prime}}}{x}\right)^{\prime}=g, \\
f^{*}(0)=f^{*}(1)=0,
\end{gathered}
$$

also has a unique solution $f^{*}(x)$, then we may define an operator

$$
T: f \longmapsto f^{*}, \quad f \in D,
$$

where $f^{*}$ is the solution of (2.5). Thus, given $Q \in[0,12.683]$, if we can prove that (3) $T$ has a fixed point, namely, there exists $f \in D$ such that $T f=f$. Then $f$ is a 
solution of (2.1). Integrating (2.1a) from 1 to $x$ and using (2.1b), we obtain (1.1a) at once, here $\beta=\left.\left[\left(f^{\prime} / x\right)^{\prime \prime}+((1+Q f) / x)\left(f^{\prime} / x\right)^{\prime}-Q\left(f^{\prime} / x\right)^{2}\right]\right|_{x=1}$. Therefore, $f$ must be the solution of (1.1).

In the following, we shall carry out the above three processes, respectively.

3. The solution of the problem (2.2). We consider the boundary value problem on $\left[x_{1}, x_{2}\right]$

$$
y^{\prime \prime}=a(x) y^{\prime}+b(x) y, \quad y\left(x_{1}\right)=A_{1}, \quad y\left(x_{2}\right)=A_{2},
$$

where $a(x), b(x) \in C^{1}\left[x_{1}, x_{2}\right]$, and $b(x)>0$.

LEMMA 3.1. Suppose that there exist functions $\bar{\omega}(x), \underline{\omega}(x) \in C^{2}\left[x_{1}, x_{2}\right]$ such that for $x_{1} \leq x \leq x_{2}$,

$$
\begin{aligned}
& \bar{\omega}(x) \geq \underline{\omega}(x), \\
& \bar{\omega}^{\prime \prime}(x) \leq a(x) \bar{\omega}^{\prime}(x)+b(x) \bar{\omega}(x), \\
& \underline{\omega}^{\prime \prime}(x) \geq a(x) \underline{\omega}^{\prime}(x)+b(x) \underline{\omega}(x), \\
& \underline{\omega}\left(x_{i}\right) \leq A_{i} \leq \bar{\omega}\left(x_{i}\right), \quad i=1,2 .
\end{aligned}
$$

Then the problem (3.1) has a unique solution $y=y(x)$, and

$$
\underline{\omega}(x) \leq y(x) \leq \bar{\omega}(x), \quad x_{1} \leq x \leq x_{2} .
$$

Moreover, there exists a positive number $N$ which depends only on the interval $\left[x_{1}, x_{2}\right]$ and the function pairs $\bar{\omega}(x), \underline{\omega}(x)$ such that

$$
\left|y^{\prime}(x)\right| \leq N, \quad x_{1} \leq x \leq x_{2} .
$$

Since $b(x)>0$, we use the maximum principle, it is easy to prove the uniqueness of solutions of (3.1), and the other aspects of Lemma 3.1 are generalizations of Nagumo's theorem (see [1, Theorem 1.5.1]).

THEOREM 3.2. Assume $f \in D$ and $0 \leq Q \leq 12.683$. Then the boundary value problem (2.2) has a unique solution $g=g(x)$.

Proof. Notice for $f \in D, x \in(0,1]$, we have

$$
\begin{aligned}
\frac{1+Q(x f)^{\prime}}{x^{2}} & =\frac{1}{x^{2}}\left[1+Q\left(x f^{\prime}+f\right)\right] \\
& \geq \frac{1}{x^{2}}[1+Q(x n(x)+h(x))] \\
& =\frac{1}{x^{2}}[1+Q F(x)],
\end{aligned}
$$

where

$$
F(x)=x n(x)+h(x)=5 x^{2}\left(\frac{1}{26} x^{26 / 5}-\frac{1}{17} x^{17 / 5}+\frac{22}{119} x^{7 / 5}-\frac{15}{119}\right) .
$$

By a simple argument, we show that $F(x)$ is decreasing on $[0, c]$, and increasing on $[c, 1]$, where $c=0.566505 \ldots$ Hence $F(x)$ takes minimum at $x=c$, that is,

$$
\min _{x \in[0,1]} F(x)=F(c)=-0.078836006 \cdots \geq-0.07884
$$


Therefore for $0 \leq Q \leq 12.683$, we have

$$
\frac{1+Q(x f)^{\prime}}{x^{2}}>0, \quad x \in(0,1]
$$

For any positive integer $n \geq 2$, consider the boundary value problem

$$
\begin{gathered}
g^{\prime \prime}=-\left[\frac{1+Q f}{x}\right] g^{\prime}+\left[\frac{1+Q(x f)^{\prime}}{x^{2}}\right] g, \\
g\left(\frac{1}{n}\right)=0, \quad g(1)=1 .
\end{gathered}
$$

We set $\underline{\omega}(x) \equiv 0, \bar{\omega}(x)=x^{\alpha}$, where $\alpha>0$ is sufficiently small, so that

$$
\alpha^{2}+\alpha Q f \leq 1+Q(x f)^{\prime} .
$$

Then

$$
\begin{aligned}
& \bar{\omega}^{\prime \prime}(x) \leq-\left[\frac{1+Q f}{x}\right] \bar{\omega}^{\prime}(x)+\left[\frac{1+Q(x f)^{\prime}}{x^{2}}\right] \bar{\omega}(x), \\
& \underline{\omega}^{\prime \prime}(x) \geq-\left[\frac{1+Q f}{x}\right] \underline{\omega}^{\prime}(x)+\left[\frac{1+Q(x f)^{\prime}}{x^{2}}\right] \underline{\omega}(x),
\end{aligned}
$$

for all $x \in[1 / n, 1], n \geq 2, \underline{\omega}(1)=0<1=\bar{\omega}(1)$, and $\underline{\omega}(1 / n)=0<1 / n^{\alpha}=\bar{\omega}(1 / n)$. By Lemma 3.1, we obtain that (3.9) has only one solution $g_{n}=g_{n}(x)$ which satisfies

$$
0 \leq g_{n}(x) \leq x^{\alpha}, \quad x \in\left[\frac{1}{n}, 1\right]
$$

and $\left\{g_{n}^{\prime}(x)\right\}$ is uniformly bounded on $[1 / 2,1]$, and hence $\left\{g_{n}^{\prime}(1)\right\}$ is bounded. Without loss of generality, we let $\left\{g_{n}^{\prime}(1)\right\} \rightarrow \alpha_{0}$ as $n \rightarrow \infty$.

We consider the solution of (3.9a) satisfying the initial conditions $g(1)=1, g^{\prime}(1)=\alpha_{0}$. Obviously, it exists on $[0,1]$ and satisfies

$$
0 \leq g(x) \leq x^{\alpha}
$$

namely, $g(x)$ is the solution of (2.2). For the uniqueness of the solutions, it is easy to show by (3.8). This completes the proof.

To prove Theorem 1.1, we give the bound of $g(x)$ and $g^{\prime}(x)$ on $[0,1]$.

THEOREM 3.3. For $f \in D, 0 \leq Q \leq 12.683$, the solution $g(x)$ of (2.2) satisfies

$$
\begin{gathered}
g(x)>0, \quad g^{\prime}(x)>0, \quad 0<x<1, \\
\lim _{x \rightarrow 0+} x g^{\prime}(x)=0, \\
x^{11 / 5} \leq g(x) \leq x^{2 / 5}, \\
\frac{2}{5} x^{11 / 5} \leq x g^{\prime}(x) \leq \frac{11}{5} x^{2 / 5} .
\end{gathered}
$$


Proof. (i) Since $1+Q(x f)^{\prime}>0, x \in[0,1]$, we easily see that $g(x)>0, g^{\prime}(x) \geq 0$ on $(0,1)$ by the maximum principle. Next we assert that $g^{\prime}(x) \neq 0$ for any $x \in(0,1)$. If not, then there exists $x_{0} \in(0,1)$ such that $g^{\prime}\left(x_{0}\right)=0$. We have $g^{\prime \prime}\left(x_{0}\right)>0$ from (2.2a), namely, $g(x)$ takes a local minimum at $x=x_{0}$. Hence $g(x)$ must have a local maximum at $x_{1} \in\left(0, x_{0}\right)$. This is impossible because we have $g^{\prime \prime}\left(x_{1}\right)>0$ from (2.2a), hence a contradiction.

(ii) Rewrite (2.2a) as follows

$$
x\left(x g^{\prime}\right)^{\prime}=-Q f g^{\prime}+\left[1+Q(x f)^{\prime}\right] g .
$$

Owing to $0 \leq x \leq 1, f \leq 0,1+Q(x f)^{\prime}>0, g>0$, we have $x\left(x g^{\prime}\right)^{\prime}>0$, that is, $\left(x g^{\prime}\right)^{\prime}>0$, and hence $x g^{\prime}$ is increasing on $(0,1)$. Using (3.14), we obtain $x g^{\prime}>0$ for $x \in(0,1)$, and therefore $\lim _{x \rightarrow 0+} x g^{\prime}(x)$ exists, and $\lim _{x \rightarrow 0+} x g^{\prime}(x) \geq 0$. If there exists $\alpha>0$ such that $\lim _{x \rightarrow 0+} x g^{\prime}(x)=\alpha$, then for $\alpha / 2$, there is a $\delta>0$, so that $\alpha / 2<x g^{\prime}(x)<g^{\prime}(1)$ for $x \in(0, \delta)$, or $\alpha / 2 x<g^{\prime}(x)<(1 / x) g^{\prime}(1)$. Integrating it from $x$ to $\delta$, we have

$$
\frac{\alpha}{2} \ln \frac{\delta}{x}<g(\delta)-g(x)<g^{\prime}(1) \ln \frac{\delta}{x} .
$$

This means $g(x) \rightarrow-\infty$ as $x \rightarrow 0+$, contradicting with $g(0)=0$. Thus $\lim _{x \rightarrow 0+} x g^{\prime}(x)=0$.

(iii) Equation (2.2a) can be converted to the following form:

$$
\left(x^{2} g^{\prime}\right)^{\prime}-(x g)^{\prime}-Q(x f g)^{\prime}=-2 Q x f g^{\prime} .
$$

Integrating the above equation from 0 to $x$, using (3.15) and $g(0)=0$, we obtain

$$
g^{\prime}=\left[\frac{1+Q f}{x}\right] g-\frac{2 Q}{x^{2}} \int_{0}^{x} t f g^{\prime} d t, \quad(0<x<1) .
$$

Hence as $-0.0406 \leq f(x) \leq 0, g^{\prime}(x) \geq 0,0 \leq Q \leq 12.683$, we have $Q f>-3 / 5$. This implies

$$
\frac{2}{5 x} g(x) \leq g^{\prime}(x) \leq \frac{1}{x} g(x)+\frac{6}{5 x} \int_{0}^{x} g^{\prime} d t=\frac{11}{5 x} g(x) .
$$

We integrate (3.22) from 1 to $x$ and obtain (3.16).

(iv) We combine (3.16) with (3.22) and yield (3.17).

This completes the proof.

4. The solution of the boundary value problem (2.5). Integrating (2.5a) from 0 to $x$ and using (2.5b), we see that

$$
\begin{gathered}
f^{*^{\prime}}(x)=k x+x \int_{0}^{x} g(t) d t, \\
f^{*}(x)=\frac{1}{2} k x^{2}+\int_{0}^{x}\left(s \int_{0}^{s} g(t) d t\right) d s,
\end{gathered}
$$

where $k=-2 \int_{0}^{1}\left(s \int_{0}^{s} g(t) d t\right) d s$. By Theorem 3.2, we know that $g(x)$ exists and is unique, so (2.5) has a unique solution $f^{*}=f^{*}(x)$ on $[0,1]$.

In the following, we estimate the bound of $f^{*}$ and $f^{* \prime}$. 
Since

$$
k=-2 \int_{0}^{1}\left(s \int_{0}^{s} g(t) d t\right) d s=-2 \int_{0}^{1}\left(g(t) \int_{t}^{1} s d s\right) d t=-\int_{0}^{1}\left(1-t^{2}\right) g(t) d t,
$$

equations (4.1) and (4.2) become

$$
\begin{gathered}
f^{*^{\prime}}(x)=-x \int_{0}^{1}\left(1-t^{2}\right) g(t) d t+x \int_{0}^{x} g(t) d t, \\
f^{*}(x)=-\frac{1}{2} x^{2} \int_{0}^{1}\left(1-t^{2}\right) g(t) d t+\frac{1}{2} \int_{0}^{x}\left(x^{2}-t^{2}\right) g(t) d t .
\end{gathered}
$$

From (4.4), we have

$$
\begin{aligned}
f^{*^{\prime}}(x) & =-x \int_{0}^{x}\left(1-t^{2}\right) g(t) d t-x \int_{x}^{1}\left(1-t^{2}\right) g(t) d t+x \int_{0}^{x} g(t) d t \\
& =-x \int_{x}^{1}\left(1-t^{2}\right) g(t) d t+x \int_{0}^{x} t^{2} g(t) d t .
\end{aligned}
$$

By (3.14) and (3.16), we have the following inequalities

$$
\begin{aligned}
f^{*^{\prime}}(x) & \leq x \int_{0}^{x} t^{2} d t=\frac{1}{3} x^{4}=n(x) \leq \frac{1}{3} \\
f^{*^{\prime}}(x) & \geq-x \int_{x}^{1}\left(1-t^{2}\right) t^{2 / 5} d t+x \int_{0}^{x} t^{2} t^{11 / 5} d t \\
& =5 x\left(\frac{1}{26} x^{26 / 5}-\frac{1}{17} x^{17 / 5}+\frac{1}{7} x^{7 / 5}-\frac{10}{119}\right) \\
& =m(x) \geq-0.0935
\end{aligned}
$$

From (4.5), it follows that

$$
f^{*}(x)=\frac{1}{2}\left(x^{2}-1\right) \int_{0}^{x} t^{2} g(t) d t-\frac{1}{2} x^{2} \int_{x}^{1}\left(1-t^{2}\right) g(t) d t .
$$

By (3.14) and (3.16), we obtain

$$
\begin{aligned}
0 & \geq f^{*}(x) \geq \frac{1}{2}\left(x^{2}-1\right) \int_{0}^{x} t^{2} t^{2 / 5} d t-\frac{1}{2} x^{2} \int_{x}^{1}\left(1-t^{2}\right) t^{2 / 5} d t \\
& =\frac{(25)}{119} x^{2}\left(x^{7 / 5}-1\right)=h(x) \geq-0.0406 .
\end{aligned}
$$

Using similar arguments, we have

$$
-0.43 \leq f^{*^{\prime \prime}}(x) \leq \frac{4}{3} .
$$

In summary, we get the following inequalities

$$
-0.0406 \leq f^{*}(x) \leq 0, \quad-0.0935 \leq f^{*^{\prime}}(x) \leq \frac{1}{3}, \quad-0.43 \leq f^{*^{\prime \prime}}(x) \leq \frac{4}{3} .
$$


5. $T$ has a fixed point. We define a norm on $C^{1}[0,1]$ by

$$
\|f\|:=\max |f|+\max \left|f^{\prime}\right|, \quad x \in[0,1] .
$$

Then $C^{1}[0,1]$ is a Banach space. It is easy to check that $D$ in Section 2 is a nonempty, closed, bounded, convex subset of $C^{1}[0,1]$. By Sections 3 and 4 , we see that $T$ is well defined and $T D \subseteq D$. In addition, $T$ maps bounded subsets of $D$ into a compact subset of $D$.

In fact, let $K \subseteq D$ be any bounded and closed subset, $\left\{f_{i}\right\} \subseteq K$ be a sequence of functions with images $\left\{f_{i}^{*}\right\} \subseteq \operatorname{Cl}(T(K))$ under $T$. Then $\left|f^{*^{\prime}}{ }_{i}\right| \leq 1 / 3$ on $[0,1]$ for each $i$. Therefore, $\left\{f_{i}^{*}\right\}$ is equicontinuous on $[0,1]$. Hence, by Arzela-Ascoli theorem, there exist a subsequence $\left\{f_{i_{k}}^{*}\right\}$ of $\left\{f_{i}^{*}\right\}$ and $g^{*} \in \mathrm{Cl}(T(K))$ such that $\left\|f_{i_{k}}^{*}-g^{*}\right\| \rightarrow 0$ as $k \rightarrow \infty$. Thus $\mathrm{Cl}(T(K))$ is compact.

Now we prove that the operator $T$ is continuous.

By the definition of $T$ and (4.2), we only need prove that for any given $f_{0} \in D$ and any $\varepsilon>0$, there exists $\delta>0$ such that as $\left\|f-f_{0}\right\|<\delta$ and $f \in D$,

$$
\max _{x \in[0,1]}\left|g(x)-g_{0}(x)\right|<\varepsilon,
$$

where $g$ and $g_{0}$ are solutions of (5.3),

$$
\begin{aligned}
& \mathfrak{g}^{\prime \prime}+\left[\frac{1+Q f}{x}\right] g^{\prime}-\left[\frac{1+Q(x f)^{\prime}}{x^{2}}\right] g=0, \quad g(0)=g(1)=0, \quad x \in[0,1] . \\
& \mathfrak{g}^{\prime \prime}+\left[\frac{1+Q f_{0}}{x}\right] g^{\prime}-\left[\frac{1+Q\left(x f_{0}\right)^{\prime}}{x^{2}}\right] g=0, \quad g(0)=g(1)=0, \quad x \in[0,1] .
\end{aligned}
$$

Let $p(x)=g(x)-g_{0}(x)$. Then by (5.3), we have

$$
\begin{aligned}
L[p] & =p^{\prime \prime}+\left[1+Q f_{0}\right] p^{\prime}-\left[\frac{1+Q\left(x f_{0}\right)^{\prime}}{x^{2}}\right] p \\
& =-\left(\frac{Q}{x^{2}}\right)\left[\left(f-f_{0}\right)\left(x g^{\prime}-g\right)-\left(f^{\prime}-f_{0}^{\prime}\right) x g\right]=-G(x),
\end{aligned}
$$

with $p(0)=p(1)=0$. For any $0 \leq x \leq \varepsilon, f \in D$ and $0 \leq Q \leq 12.683$, using (3.16), we obtain

$$
|p(x)|=\left|g(x)-g_{0}(x)\right| \leq 2 x^{2 / 5} \leq 2 \varepsilon^{2 / 5}=\varepsilon_{1} .
$$

For $x \in[\varepsilon, 1],\left\|f-f_{0}\right\| \rightarrow 0$ and $0 \leq Q \leq 12.683$, we claim that $|p(x)|<\varepsilon_{1}$.

Set

$$
\varepsilon^{*}=(1-0.07884 Q) \varepsilon_{1} .
$$

If $\left\|f-f_{0}\right\|$ is sufficient by small, $x \in[\varepsilon, 1]$ and $0 \leq Q \leq 12.683$, then by (3.16) and (3.17), we have

$$
\begin{aligned}
|G(x)| & \leq \frac{Q}{\varepsilon^{2}}\left[\max \left\{\left|x g^{\prime}\right|+|x g|+|g|\right\}\right]\left\|f-f_{0}\right\| \\
& \leq \frac{Q}{\varepsilon^{2}}\left(\frac{11}{5}+1+1\right)\left\|f-f_{0}\right\| \\
& =\frac{21 Q}{5 \varepsilon^{2}}\left\|f-f_{0}\right\|<\varepsilon^{*} .
\end{aligned}
$$


Fix $f_{0}$, let $p$ be a solution of (5.4) with the boundary conditions $|p(\varepsilon)| \leq \varepsilon_{1}$ and $p(1)=$ 0 . By (5.7), we have

$$
L[p]-\varepsilon^{*} \leq L[p]+F(x) \leq L[p]+\varepsilon^{*}, \quad(\varepsilon \leq x \leq 1) .
$$

Let $p^{ \pm}$be the solutions of the following problems

$$
L[p] \pm \varepsilon^{*}=0, \quad p^{ \pm}(1)=0, \quad p^{ \pm}(\varepsilon)=p(\varepsilon) .
$$

Then using the comparison theorem, we show that

$$
p^{-}(x) \leq p(x) \leq p^{+}(x), \quad x \in[\varepsilon, 1] .
$$

Now we prove that for any $x \in[\varepsilon, 1]$, there is $p^{+}(\varepsilon) \leq \varepsilon_{1}$. In fact, we see that $p^{+}(x) \leq \varepsilon_{1}$ as $|p(\varepsilon)| \leq \varepsilon_{1}$. If not, then there exists a point $x_{+} \in(\varepsilon, 1)$ such that $p^{+}\left(x_{+}\right)>\varepsilon_{1}$. By $p^{+}(\varepsilon)=p(\varepsilon) \leq \varepsilon_{1}$, there must be a point $y_{+} \in(\varepsilon, 1)$, such that $p^{+}(x)$ takes maximum at $y_{+}$, namely

$$
p^{+}\left(y_{+}\right)>\varepsilon_{1}, \quad p^{+\prime}\left(y_{+}\right)=0, \quad p^{+\prime \prime}\left(y_{+}\right)<0 .
$$

But by (5.6) and (5.11), we see that the following hold

$$
p^{+\prime}\left(y_{+}\right)+\varepsilon^{*}=\left[\frac{1+Q f_{0}\left(y_{+}\right)+Q f_{0}^{\prime}\left(y_{+}\right) y_{+}}{y_{+}^{2}}\right] p^{+}\left(y_{+}\right)>(1-0.07884 Q) \varepsilon_{1} .
$$

Hence, using (5.6), we get

$$
p^{+\prime \prime}\left(y_{+}\right)>(1-0.07884 Q) \varepsilon_{1}-\varepsilon^{*}=0,
$$

a contradiction. For $x \in[\varepsilon, 1]$ and $|p(\varepsilon)| \leq \varepsilon_{1}$, we argue similarly and obtain $p^{-}(x) \geq$ $-\varepsilon_{1}$. Thus, when $x \in[\varepsilon, 1]$ and $|p(\varepsilon)| \leq \varepsilon_{1}$, there be $|p(x)| \leq \varepsilon_{1}$.

Therefore, for any given $\varepsilon>0$, if we choose

$$
\delta=\frac{\varepsilon^{*}}{21 Q / 5 \varepsilon^{2}},
$$

where $\varepsilon^{*}$ satisfies (5.6), then for $f \in D$, $\max _{x \in[0,1]}\left|g(x)-g_{0}(x)\right|=\varepsilon_{1}=2 \varepsilon^{2 / 5}$ as $\left\|f-f_{0}\right\|<\delta$, and the continuity of $T$ is proved.

To sum up, we see that the operator $T$ satisfies the conditions of Schauder's fixed point theorem [2], and thus $T$ has at least one fixed point in $D$.

By Sections 2, 3, 4, and 5, Theorem 1.1 is proved.

\section{REFERENCES}

[1] S. R. Bernfeld and V. Lakshmikantham, An Introduction to Nonlinear Boundary Value Problems, vol. 109, Academic Press, [A subsidiary of Harcourt Brace Jovanovich, Publishers], New York, 1974, Mathematics in Science and Engineering. MR 56\#3393. Zbl 286.34018.

[2] K. Deimling, Nonlinear Functional Analysis, Springer-Verlag, Berlin, 1985. MR 86j:47001. Zbl 559.47040 .

[3] W. N. Gill, N. D. Kazarinoff, C. Hus, M. Noack, and J. D. Verhoeven, Thermo-capillary driven convection in supported and floating zone crystallization, Adv. in Space Res. 4 (1984), $15-22$. 
[4] C. Q. Lu and N. D. Kazarinoff, On the existence of solutions of a two-point boundary value problem arising from flows in a cylindrical floating zone, SIAM J. Math. Anal. 20 (1989), no. 2, 494-503. MR 90b:34025. Zbl 689.34016.

Shi Yongdong: Research Centre of Financial Engineering, Department of Finance, DONGBEI UNIVERSITY OF FINANCE AND ECONOMICS, BOX 74, DALIAN 116025, CHINA

E-mail address: shiyd@on 7 ine. $7 n . c n$

Du LiAngSheng: Department of EConomics, Dongbei University of FinANCE AND ECONOMICS, BOX 74, DALIAN 116025, CHINA 


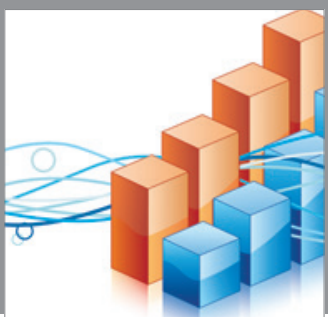

Advances in

Operations Research

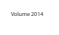

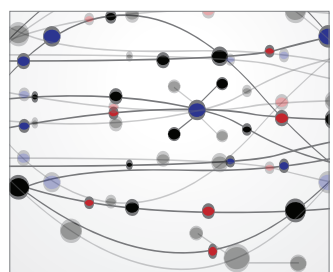

\section{The Scientific} World Journal
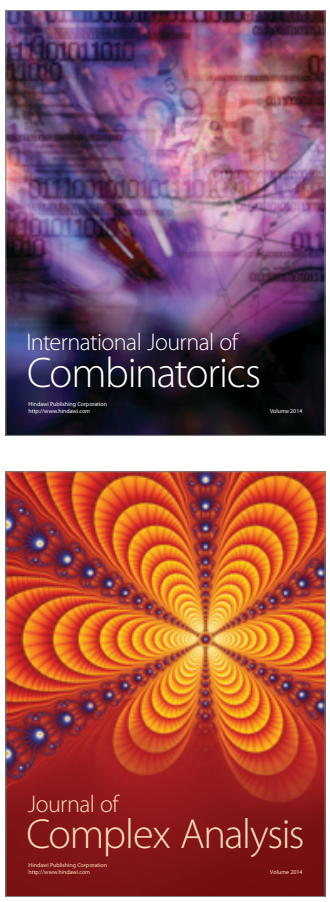

International Journal of

Mathematics and

Mathematical

Sciences
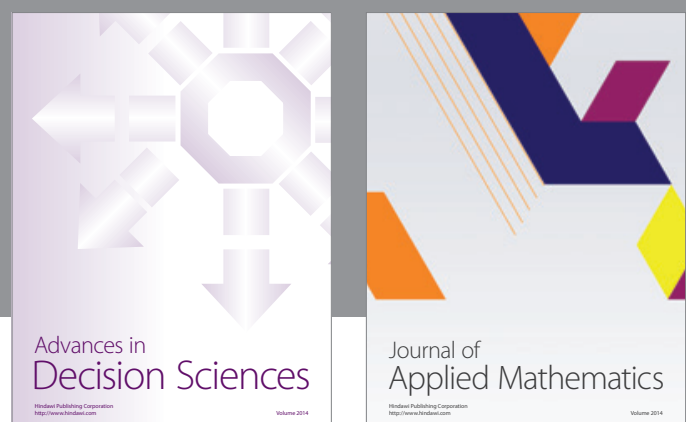

Journal of

Applied Mathematics
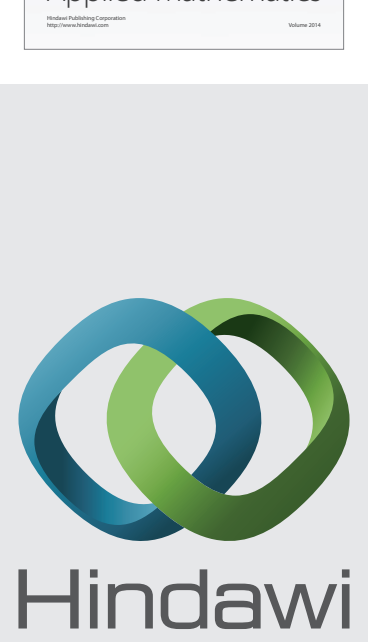

Submit your manuscripts at http://www.hindawi.com
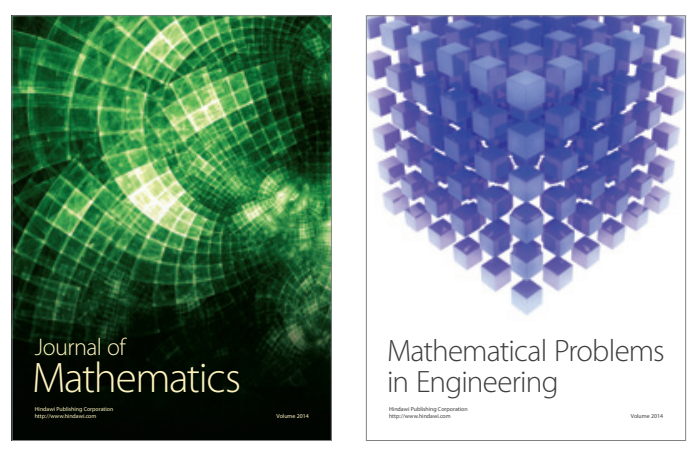

Mathematical Problems in Engineering
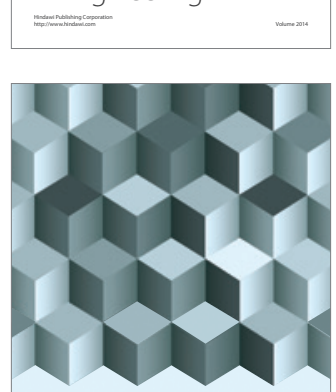

Journal of

Function Spaces
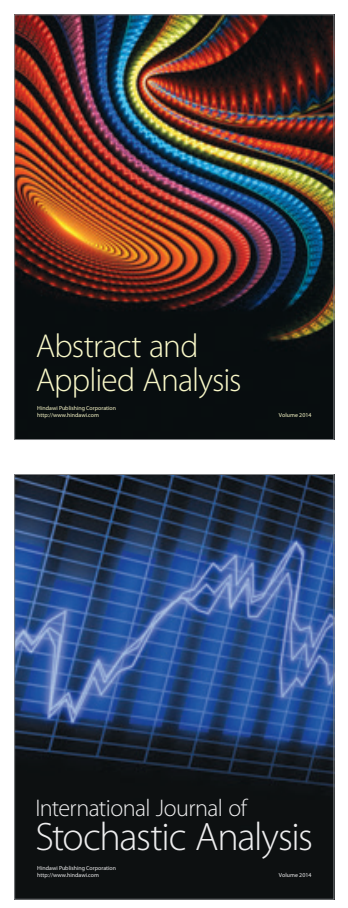

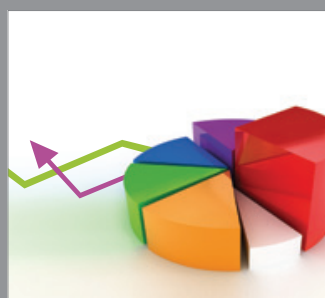

ournal of

Probability and Statistics

Promensencen
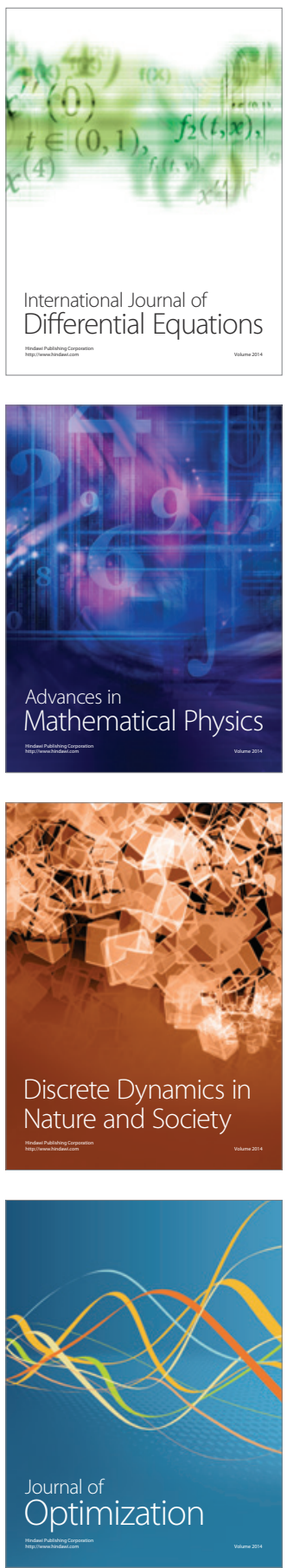